\title{
Force and Torque Analytical Models of a Reaction Sphere Actuator Based on Spherical Harmonic Rotation and Decomposition
}

\author{
Leopoldo Rossini, Olivier Chételat, Emmanuel Onillon, and Yves Perriard
}

\begin{abstract}
This paper presents an analytical model for the force and torque developed by a reaction sphere actuator for satellite attitude control. The reaction sphere is an innovative momentum exchange device consisting of a magnetic bearings spherical rotor that can be electronically accelerated in any direction making all the three axes of stabilized spacecrafts controllable by a unique device. The spherical actuator is composed of an 8-pole permanent magnet spherical rotor and of a 20 -coil stator. Force and torque analytical models are derived by solving the Laplace equation and applying the Lorentz force law. The novelty consists in exploiting powerful properties of spherical harmonic functions under rotation to derive closed-form linear expressions of forces and torques for all possible orientations of the rotor. Specifically, the orientation of the rotor is parametrized using seven decomposition coefficients that can be determined noniteratively and in a linear fashion by measuring the radial component of the magnetic flux density from at least seven different locations. Therefore, force and torque models for all possible orientations of the rotor are expressed in closed form as linear combination of mutually orthogonal force and torque characteristic matrices, which are computed offline. The proposed analytical models are experimentally validated using a developed laboratory prototype.
\end{abstract}

Index Terms-Force and torque model, reaction sphere, satellite attitude control, spherical actuator.

\section{INTRODUCTION}

A TTITUDE and orbit control systems (AOCS) are responsible for the orbital behavior and pointing precision of stabilized satellites and have a major impact on the efficiency and quality of commercial and scientific space missions [1]. AOCS require a minimum of three reaction wheels (RW) or control moment gyroscopes (CMG), but in practice, four or five wheels are common for optimization and redundancy purposes.

Manuscript received July 1, 2011; revised November 3, 2011 and February 26, 2012; accepted April 5, 2012. Date of publication May 9, 2012; date of current version January 18, 2013. Recommended by Technical Editor G. Herrmann. This work was supported in part by the European Space Agency (ESA) through GSTP Contract 20227/06/NL/SFe and NPI Contract 40000101313/10/NL/PA, and in part by maxon motor ag.

L. Rossini, O. Chételat, and E. Onillon are with the Systems Division of the Swiss Center for Electronics and Microtechnology, CH-2000 Neuchâtel, Switzerland (e-mail: leopoldo.rossini@csem.ch; olivier.chetelat@csem.ch; emmanuel.onillon@csem.ch).

Y. Perriard is with the Integrated Actuators Laboratory, Ecole Polytechnique Federale de Lausanne, Lausanne 1015, Switzerland (e-mail: yves. perriard@epfl.ch).

Color versions of one or more of the figures in this paper are available online at http://ieeexplore.ieee.org.

Digital Object Identifier 10.1109/TMECH.2012.2195501

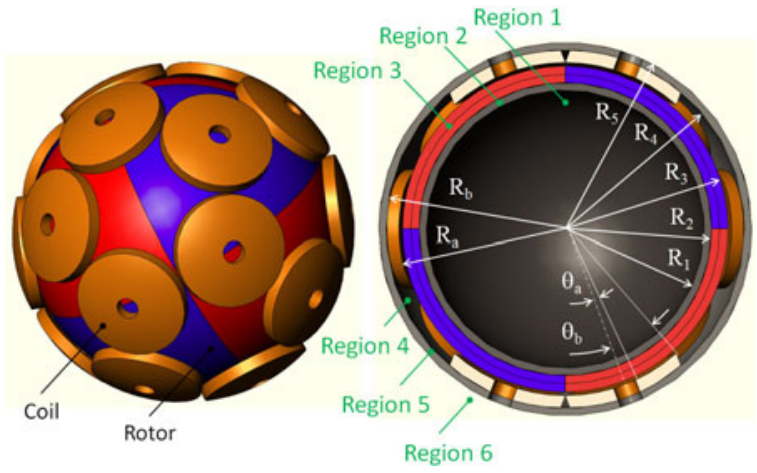

Fig. 1. Schematic illustration of the 3-D motor of the reaction sphere, which is composed of an 8-pole rotor (cube) and a 20-pole stator (dodecahedron). For illustrative purposes, the iron stator is not shown in the left figure.

The attitude of the satellite can be changed by reaction to the acceleration of the appropriate wheel [2].

We propose to employ a single-reaction sphere held in position by magnetic levitation as an alternative to traditional RW or CMG approaches. The sphere can be accelerated in any direction by a 3-D motor making all the three axes of the spacecraft controllable by just a single device. Furthermore, the reaction sphere rotor being magnetically levitated, the absence of friction improves the pointing performance of the satellite with respect to conventional ball bearing momentum exchange devices. The proposed approach is patent protected [3]. A schematic representation of this spherical actuator is presented in Fig. 1. The 3-D motor is synchronous and the novelty consists in arranging permanent magnets and electromagnets at locations following the vertices of regular polygons. The permanent magnet motor is composed of a rotor with eight poles, each of them being either a north pole if $x y z>0$ or a south pole otherwise $(x, y$, and $z$ are the coordinates of a given point of the sphere). The permanent magnets (region 3 ) are adjusted on a back iron hollow spherical shell (region 2). The stator has 20 air-core electromagnets, each corresponding to one vertex of a dodecahedron. The electromagnets are mounted on a ferromagnetic spherical support (region 5). Notice that a ferromagnetic stator increases the actuator forces and torques and at the same time provides an important shielding role [4].

The proposed reaction sphere can be classified under the family of electromagnetic spherical actuators. A variety of different electromagnetic spherical motors, mainly for robotic purposes, have been proposed in the literature [5]-[9]. However, these design concepts and developed prototypes share some common 
application-driven features that, to our understanding, prevent them from being employed as attitude control actuators. First, a mechanical shaft emerges from the rotor to export the torque, breaking the mechanical symmetry of the designs and limiting the tilting range to a maximum of $\pm 45^{\circ}$. Conversely, as in the aimed application the torque is exported to the satellite by conservation of the angular momentum [2], the structure of the proposed reaction sphere is symmetric with no physical constraints on the rotation. Furthermore, differently from [5], [6], and [8], where the rotor of the presented actuators is supported by either low-friction surface coating or by, respectively, hydrostatic and aerostatic bearings, the rotor of the reaction sphere is magnetically levitated and no friction occurs during operation.

Force and torque direct models are necessary for the operation of spherical actuators as they allow relating a given set of stator currents to force and torque outputs. Depending on the architecture of the design, force and torque direct models between stator coils and the permanent magnet rotor are obtained with multiple approaches. Finite element simulations to compute force characteristics between a stator coil and the rotor of a spherical machine with variable pole pitch were proposed in [6]. The torque output of a spherical actuator consisting of a spherical rotor with dihedral-shaped permanent magnets and multiple layer stator coils was derived by solving the Laplace equation and employing Lorentz integration in [7]. An alternative approach to characterize torque models, while reducing the computational time, consists in modeling both the stator coils and the permanent magnets as distributed multipoles (DMP). Unlike the Lorentz force or the Maxwell stress tensor methods that require integration, the dipole force computation is expressed in closed form [10]. For the proposed reaction sphere actuator, similarly to [11], force and torque models were derived by solving the Laplace equation and subsequently applying the Lorentz force law [12].

To our understanding, a common characteristic of these modeling techniques is that force and torque models are intrinsically dependent on the orientation of the rotor. Thus, force and torque calculations are carried out by applying a change of coordinates in the derived magnetic flux models, generally parameterized with a set of three Euler angles to account for the orientation of the rotor. To this end, several noncontact-based methods were proposed to measure the orientation of the spherical rotor. For instance, a vision-based approach combined with a recursive nonlinear optimization algorithm was proposed in [13]. However, the nonlinear nature of the problem to be solved requires a fairly good initial guess of the orientation. In [14], the authors proposed a laser-based-orientation measurement but the apparatus requires a flat reflecting plate that cannot be embedded in our design. An optimization strategy for position and orientation tracking of moving objects in 3-D space is proposed in [15]. The method is based on a distributed multiple pole model of the moving object but requires three-axis Hall sensors. Another proposed approach consists of a direct method that maps distributed two-axis and three-axis magnetic flux density measurements to the instantaneous orientation of the rotor [16]. The proposed mapping is based on an artificial neural network.
In this paper, we propose the following.

1) A development of force and torque analytical models for the reaction sphere actuator that are intimately related to the rotor-orientation measurement. In particular, thanks to the proposed magnetization of the permanent magnet rotor, the development of the magnetic flux density analytical model is carried out in stator coordinates exploiting powerful properties of spherical harmonics under rotation. The resulting models are linear and relate a set of stator currents to the output forces and torques. The novelty consists in parameterizing the analytical model using seven spherical harmonic decomposition coefficients that provide an analytical expression of the magnetic flux density for any possible orientation of the rotor.

2) An estimation method of the seven spherical harmonic decomposition coefficients. Conversely from [16], where two-axis and three-axis magnetic flux density measurements are necessary, the proposed estimation method is based on measuring the radial component of the magnetic flux density at multiple locations equidistant from the rotor surface. Moreover, differently from [17], where the radial component needs to be measured on all the boundary surface, the magnetic flux density in the airgap can be reconstructed with only $N \geq 7$ measurements. Finally, contrarily to the estimation of a set of three Euler angles in [13], the proposed procedure is linear and it is expressed in closed form.

3) A step by step implementation procedure of the proposed force and torque models.

4) A numerical verification using finite element simulations, where stator currents are computed to satisfy reference forces and torques for the rotor about several randomly generated orientations.

5) An experimental verification of the proposed models using the laboratory prototype we have developed.

\section{Magnetic FluX Density Model}

In this section, an analytical expression for the magnetic flux density in the airgap of the reaction sphere is developed solving Laplace's and Poisson's equations. The development of the magnetic flux density model follows our previous work presented in [12] with a significant variation introduced to express the field of a rotated rotor in stator coordinates.

\section{A. Constitutive Relations}

For the formulation of the analytical model, we use the rotor and stator model reported in Fig. 1 with the respective relevant dimensions. The reaction sphere is composed of a rotor back iron (region 2) a permanent magnet (region 3), an airgap that includes the coils (region 4), and an iron stator (region 5). The rotor back iron is hollow (region 1) and the reaction sphere is surrounded by air (region 6). 
The constitutive relations describing the magnetic effects inside the six regions are characterized by

$$
\mathbf{B}_{i}=\left[\begin{array}{c}
B_{i r} \\
B_{i \theta} \\
B_{i \phi}
\end{array}\right]=\left\{\begin{array}{l}
\mu_{0} \mathbf{H}_{i}, \text { for } i=1,4,6 \\
\mu_{0} \mu_{r} \mathbf{H}_{i}, \text { for } i=2,5 \\
\mu_{0} \mu_{m} \mathbf{H}_{i}+\mathbf{B}_{\mathrm{r}}, \text { for } i=3
\end{array}\right.
$$

where the subscript $i$ denotes the region numbers; $\mathbf{B}$ and $\mathbf{H}$ are the magnetic flux density and the magnetic field, respectively; $\mu_{0}$ is the vacuum magnetic permeability, while $\mu_{r}$ and $\mu_{m}$ are the magnetic permeability of the iron and the permanent magnet material, respectively. Finally, $\mathbf{B}_{\mathrm{r}}$ is the remanence of the permanent magnet.

\section{B. Rotor Magnetization}

In order to obtain an 8-pole spherical rotor, the following remanence is applied to the permanent magnet of an immobile rotor:

$$
\mathbf{B}_{\mathrm{r}, \mathrm{imm}}=\left[\begin{array}{c}
B_{\mathrm{r} r} \\
B_{\mathrm{r} \theta} \\
B_{\mathrm{r} \phi}
\end{array}\right]=\left[\begin{array}{c}
\frac{Y_{3}^{2}(\theta, \phi)-Y_{3}^{-2}(\theta, \phi)}{Y_{3}^{2}\left(\theta_{p}, \phi_{p}\right)-Y_{3}^{-2}\left(\theta_{p}, \phi_{p}\right)} \\
0 \\
0
\end{array}\right] B_{\mathrm{r}}
$$

where $B_{\mathrm{r}}$ is the constant magnetic remanence modulated by the complex spherical harmonics $Y_{3}^{-2}$ and $Y_{3}^{2}$, while $\theta_{p}=$ $\cos ^{-1}(1 / \sqrt{3})$ and $\phi_{p}=\pi / 4$ are the coordinates defining a pole. We refer the reader to Appendix for a brief overview on spherical harmonics. To calculate the radial magnetization of a rotated rotor expressed in stator coordinates, the radial component of the magnetization of the immobile rotor in (2) is decomposed in terms of spherical harmonics of degree 3 as

$$
B_{\mathrm{r} r}(\theta, \phi)=B_{\mathrm{r}} \sum_{m=-3}^{3} c_{3, \mathrm{imm}}^{m} Y_{3}^{m}(\theta, \phi)
$$

where, by the orthogonality property of spherical harmonics, the decomposition coefficients $c_{3, \mathrm{imm}}^{m}$ for the immobile rotor can be expressed as

$$
c_{3, \mathrm{imm}}^{m}= \begin{cases} \pm \frac{1}{Y_{3}^{2}\left(\theta_{p}, \phi_{p}\right)-Y_{3}^{-2}\left(\theta_{p}, \phi_{p}\right)}, & \text { if } m= \pm 2 \\ 0, & \text { otherwise. }\end{cases}
$$

The radial magnetization of a rotated rotor expressed in terms of stator coordinates can be calculated by considering the effect that a rotation operator $R(\alpha, \beta, \gamma)$, parameterized using $\mathrm{ZYZ}$ Euler angles $\alpha, \beta$, and $\gamma$, has on the spherical harmonic decomposition coefficients $c_{3, \mathrm{imm}}^{m}$ of degree 3 . The effect of such a rotation can be formulated as

$$
c_{3}^{m}(\alpha, \beta, \gamma)=\sum_{n} D_{m n}^{3}(\alpha, \beta, \gamma) c_{3, \mathrm{imm}}^{n}
$$

where $D_{m n}^{3}(\alpha, \beta, \gamma)$ are unitary rotation matrices [18]. Hence, the spherical harmonic basis functions are decomposed into a direct sum of orthogonal subspaces that are globally invariant under rotation. Therefore, as the decomposition coefficients of the immobile rotor are nonzero for $n= \pm 2$, the radial magnetization of a rotated rotor expressed in stator coordinates can be calculated as

$$
B_{\mathrm{r} r}(\theta, \phi)=B_{\mathrm{r}} \sum_{m=-3}^{3} c_{3}^{m} Y_{3}^{m}(\theta, \phi)
$$

where

$$
c_{3}^{m}=D_{m, 2}^{3}(\alpha, \beta, \gamma) c_{3, \mathrm{imm}}^{2}+D_{m,-2}^{3}(\alpha, \beta, \gamma) c_{3, \mathrm{imm}}^{-2} .
$$

Finally, using (6), the magnetic remanence for any possible orientation of the rotor can be formulated as

$$
\mathbf{B}_{\mathrm{r}}=\left[\begin{array}{c}
B_{\mathrm{r} r} \\
B_{\mathrm{r} \theta} \\
B_{\mathrm{r} \phi}
\end{array}\right]=\left[\begin{array}{c}
B_{\mathrm{r}} \sum_{m=-3}^{3} c_{3}^{m} Y_{3}^{m}(\theta, \phi) \\
0 \\
0
\end{array}\right] .
$$

Therefore, the remanence of a rotated rotor is function of the spherical harmonic decomposition coefficients $c_{3}^{ \pm 3}, c_{3}^{ \pm 2}, c_{3}^{ \pm 1}$, and $c_{3}^{0}$. However, since $c_{n}^{-m}=(-1)^{m} \overline{c_{n}^{m}}$, where $\overline{c_{n}^{m}}$ denotes the complex conjugate of $c_{n}^{m}$, the radial magnetization of the rotor is parameterized using seven real coefficients only. Finally, notice that the physical orientation of the rotor in terms of Euler angles can be determined from unitary matrices $D_{m, \pm 2}^{3}$ as already proposed, for instance, in a computer vision application [19].

\section{Governing Equations}

Starting from the magnetostatic field equation for currentfree regions $\nabla \times \mathbf{H}=0$, the magnetic field $\mathbf{H}$ in region $i$ is calculated as the gradient of a magnetic scalar potential $\varphi$ [20]

$$
\mathbf{H}_{i}=\left[\begin{array}{c}
H_{i r} \\
H_{i \theta} \\
H_{i \phi}
\end{array}\right]=-\nabla \varphi_{i}=\left[\begin{array}{c}
-\frac{\partial \varphi_{i}}{\partial r} \\
-\frac{1}{r} \frac{\partial \varphi_{i}}{\partial \theta} \\
-\frac{1}{r \sin \theta} \frac{\partial \varphi_{i}}{\partial \phi}
\end{array}\right] .
$$

Then, substituting the constitutive relations in (1) into $\nabla \cdot \mathbf{B}=$ 0 , and employing (9), we obtain the Laplace equations for regions $i=1,2,4,5,6$

$$
\nabla^{2} \varphi_{i}=0
$$

and the Poisson equation for the permanent magnet in region $i=3$

$$
\nabla^{2} \varphi_{3}=\frac{1}{\mu_{0} \mu_{m}} \nabla \cdot \mathbf{B}_{\mathrm{r}} .
$$

With definition (8), the Poisson equation (11) for the permanent magnet can be rewritten as

$$
\nabla^{2} \varphi_{3}=\frac{2}{\mu_{0} \mu_{m}} \frac{1}{r} B_{\mathrm{r}} \sum_{m=-3}^{3} c_{3}^{m} Y_{3}^{m}(\theta, \phi) .
$$

The solutions $\varphi_{i}$ of (10) and (12) are the magnetic scalar potentials inside the six regions. Once these equations are solved, the magnetic flux density within region $i$ can be calculated by 
first employing definition (9) and subsequently applying the respective constitutive relations (1).

\section{General Solution to Laplace's and Poisson's Equations}

The general solution of the Laplace equation in spherical coordinates characterizing region $i=1,2,4,5,6$ is [11]

$$
\varphi_{i}=\sum_{n=0}^{\infty} \sum_{m=-n}^{n}\left(\kappa_{n, i}^{m} r^{n}+\xi_{n, i}^{m} r^{-(n+1)}\right) Y_{n}^{m}(\theta, \phi)
$$

where $\kappa_{n, i}^{m}$ and $\xi_{n, i}^{m}$ are coefficients to be defined using boundary conditions while $Y_{n}^{m}(\theta, \phi)$ are complex-valued spherical harmonic functions. The general solution to the Poisson equation (12) is obtained by including an additional term to solution (13) so as to satisfy the conditions imposed by the excitation term on the right-hand side of (12). Therefore

$$
\begin{aligned}
\varphi_{3}=\sum_{n=0}^{\infty} \sum_{m=-n}^{n}\left(\kappa_{n, 3}^{m} r^{n}+\xi_{n, 3}^{m} r^{-(n+1)}\right) Y_{n}^{m}(\theta, \phi) \\
-\frac{r}{5 \mu_{0} \mu_{m}} B_{\mathrm{r}} \sum_{m=-3}^{3} c_{3}^{m} Y_{3}^{m}(\theta, \phi) .
\end{aligned}
$$

\section{E. Boundary Conditions}

A set of boundary conditions is necessary to determine coefficients $\kappa_{n, i}^{m}$ and $\xi_{n, i}^{m}, i=1,2, \ldots, 6$, that provide particular solutions to (10) and (12). Boundary conditions for the reaction sphere configuration can be summarized as

$$
\begin{aligned}
& \left.B_{6 r}\right|_{r \rightarrow \infty}=0,\left.\quad B_{6 \theta}\right|_{r \rightarrow \infty}=0,\left.\quad B_{6 \phi}\right|_{r \rightarrow \infty}=0 \\
& \left.B_{1 r}\right|_{r=0} \neq \infty,\left.\quad B_{1 \theta}\right|_{r=0} \neq \infty,\left.\quad B_{1 \phi}\right|_{r=0} \neq \infty \\
& \left.B_{i r}\right|_{r=R_{i}}=\left.B_{i+1 r}\right|_{r=R_{i}} \text { and } \\
& \left.H_{i \theta}\right|_{r=R_{i}}=\left.H_{i+1 \theta}\right|_{r=R_{i}},\left.\quad H_{i \phi}\right|_{r=R_{i}}=\left.H_{i+1 \phi}\right|_{r=R_{i}} .
\end{aligned}
$$

Expressions (15) and (16) are the boundary conditions to be satisfied at the far field and at the origin, where the magnetic flux density approaches zero when $r \rightarrow \infty$ and must be finite at $r=0$. Furthermore, boundary conditions (17) specify that the radial component of $\mathbf{B}$ must be continuous across the interface $i$ and $i+1$. Finally, conditions (18) indicate that, in absence of a free surface current density, the tangential component of $\mathbf{H}$ must also be continuous at the interface [20].

\section{F. Solution}

Forces and torques are produced by the interaction of the magnetic flux density with the stator coils located in region 4. Hence, only the magnetic scalar potential $\varphi_{4}$ within the airgap will be calculated. Invoking the orthogonal property of spherical harmonics, using the definition in (9) together with general solutions (13) and (14), boundary conditions (15)-(18) result in

$$
\left\{\begin{array}{l}
\kappa_{3,6}^{m}=0 \\
\xi_{3,1}^{m}=0 \\
\kappa_{3,1}^{m} R_{1}^{3}-\kappa_{3,2}^{m} R_{1}^{3}-\xi_{3,2} R_{1}^{-4}=0 \\
\kappa_{3,2}^{m} R_{2}^{3}+\xi_{3,2} R_{2}^{-4}-\kappa_{3,3}^{m} R_{2}^{3}-\xi_{3,3} R_{2}^{-4}=0 \\
\kappa_{3,3}^{m} R_{3}^{3}+\xi_{3,3} R_{3}^{-4}-\kappa_{3,4}^{m} R_{3}^{3}-\xi_{3,4} R_{3}^{-4}=0 \\
\kappa_{3,4}^{m} R_{4}^{3}+\xi_{3,4} R_{4}^{-4}-\kappa_{3,5}^{m} R_{4}^{3}-\xi_{3,5} R_{4}^{-4}=0 \\
\kappa_{3,5}^{m} R_{5}^{3}+\xi_{3,5} R_{5}^{-4}-\xi_{3,6} R_{5}^{-4}=0 \\
3 \kappa_{3,1}^{m} R_{1}^{2}-3 \mu_{r} \kappa_{3,2}^{m} R_{1}^{2}+4 \mu_{r} \xi_{3,2}^{m} R_{1}^{-5}=0 \\
3 \mu_{r} \kappa_{3,2}^{m} R_{2}^{2}-4 \mu_{r} \xi_{3,2}^{m} R_{2}^{-5}-3 \mu_{m} \kappa_{3,3}^{m} R_{2}^{2}+4 \mu_{m} \xi_{3,3}^{m} R_{2}^{-5} \\
\quad \quad+6 B_{\mathrm{r}} / \mu_{0} c_{3}^{m}=0 \\
3 \mu_{m} \kappa_{3,3}^{m} R_{3}^{2}-4 \mu_{m} \xi_{3,3}^{m} R_{3}^{-5}-6 B_{\mathrm{r}} / \mu_{0} c_{3}^{m}-3 \kappa_{3,4}^{m} R_{3}^{2} \\
\quad \quad+4 \xi_{3,4}^{m} R_{3}^{-5}=0 \\
3 \kappa_{3,4}^{m} R_{4}^{2}-4 \xi_{3,4}^{m} R_{4}^{-5}-3 \mu_{r} \kappa_{3,5}^{m} R_{4}^{2}+4 \mu_{r} \xi_{3,5}^{m} R_{4}^{-5}=0 \\
3 \mu_{r} \kappa_{3,5}^{m} R_{5}^{2}-4 \mu_{r} \xi_{3,5}^{m} R_{5}^{-5}+4 \xi_{3,6}^{m} R_{5}^{-5}=0
\end{array}\right.
$$

that is a system of 12 linear equations for the 12 unknown coefficients $\kappa_{3, i}^{m}$ and $\xi_{3, i}^{m}, i=1,2, \ldots, 6$. Defining

$$
K_{1}=\frac{\xi_{3,4}^{m}}{B_{\mathrm{r}} c_{3}^{m}} \mu_{0}, \quad K_{2}=\frac{\kappa_{3,4}^{m}}{B_{\mathrm{r}} c_{3}^{m}} \mu_{0}
$$

and solving the system of linear equations (19) for the desired coefficients $\kappa_{3,4}^{m}$ and $\xi_{3,4}^{m}$, (13) for the magnetic scalar potential within the airgap can be rewritten as

$$
\varphi_{4}=r^{-4}\left(K_{1}+K_{2} r^{7}\right) \frac{B_{\mathrm{r}}}{\mu_{0}} \sum_{m=-3}^{3} c_{3}^{m} Y_{3}^{m}(\theta, \phi)
$$

where $K_{1}$ and $K_{2}$ are constant. Although easily calculated using a program of symbolic calculation, expressions for $K_{1}$ and $K_{2}$ are excessively long for a finite value of the permeability $\mu_{r}$. Therefore, we will assume that the iron is infinitely permeable $\left(\mu_{r} \rightarrow \infty\right)$. Notice that with this assumption, the number of conditions in (19) reduces to 4 and coefficients $\kappa_{3, i}^{m}=0$ and $\xi_{3, i}^{m}=0, \forall i \neq 3,4$. Therefore,

$$
K_{1}=\frac{R_{3}^{5}}{5} \frac{\hat{K}_{1}}{\check{K}_{1}} \quad K_{2}=-K_{1} R_{4}^{-7}
$$

with

$$
\hat{K}_{1}=\frac{\left(1+3 / 4 R_{2}^{-7} R_{3}^{7}\right)\left(1-R_{2}^{-2} R_{3}^{2}\right)}{\left(1+3 / 4 R_{4}^{-7} R_{3}^{7}\right)\left(1-R_{2}^{-7} R_{3}^{7}\right)}+\frac{3 / 2\left(1-1 / 2 R_{2}^{-2} R_{3}^{2}\right)}{\left(1+3 / 4 R_{4}^{-7} R_{3}^{7}\right)}
$$

and

$$
\check{K}_{1}=1-\mu_{m} \frac{\left(1+3 / 4 R_{2}^{-7} R_{3}^{7}\right)\left(1-R_{4}^{-7} R_{3}^{7}\right)}{\left(1+3 / 4 R_{4}^{-7} R_{3}^{7}\right)\left(1-R_{2}^{-7} R_{3}^{7}\right)} .
$$

Then, the magnetic scalar potential (21) becomes

$$
\varphi_{4}=r^{-4}\left(1-R_{4}^{-7} r^{7}\right) \frac{K_{1} B_{\mathrm{r}}}{\mu_{0}} \sum_{m=-3}^{3} c_{3}^{m} Y_{3}^{m}(\theta, \phi) .
$$


Finally, the magnetic flux density in the airgap is calculated using (9) and the constitutive relation in air $\mathbf{B}_{4}=\mu_{0} \mathbf{H}_{4}$ as

$$
\mathbf{B}_{4}=K_{1} B_{\mathrm{r}} \sum_{m=-3}^{3} c_{3}^{m} \nabla\left[r^{-4}\left(R_{4}^{-7} r^{7}-1\right) Y_{3}^{m}(\theta, \phi)\right]
$$

that can also be expressed as the sum of the magnetic flux densities $\mathbf{B}_{4}^{m}$ generated by each spherical harmonic of degree 3 and order $m$ as

$$
\mathbf{B}_{4}=\sum_{m=-3}^{3} c_{3}^{m} \mathbf{B}_{4}^{m}
$$

where

$$
\mathbf{B}_{4}^{m}=K_{1} B_{\mathrm{r}} \nabla\left[r^{-4}\left(R_{4}^{-7} r^{7}-1\right) Y_{3}^{m}(\theta, \phi)\right] .
$$

\section{FORCE AND TORQUE MODELS}

Force and torque models are necessary for the design of closed-loop dynamic controllers. On one hand, the force is required to control the position of the rotor inside the stator. On the other hand, the torque allows controlling the rotation of the rotor so as to influence the attitude of the satellite. Force and torque models are derived by first considering the interaction between the rotor magnetic flux density and one stator coil and by subsequently invoking the superposition principle to calculate forces and torques generated by the complete set of coils.

\section{A. Force Generated by a Coil}

To calculate the force produced by a single coil, we consider the latter as a spherical portion delimited by angles $\theta_{a}$ and $\theta_{b}$, and by the spherical radii $R_{a}$ and $R_{b}$ as illustrated in Fig. 1 . The position of the coil in the stator frame $[X, Y, Z]$ can be described by the rotation matrix $\tilde{R}(\alpha, \beta)$ that is parametrized using angles $\alpha$ and $\beta$ that completely specify its location. As the Lorentz force integration will be carried out in Cartesian coordinates, the flux density (26) is expressed in these coordinates as $\mathbf{B}_{4}(x, y, z)$. This expression can be derived from (26) by using the transformation $x=r \sin \theta \cos \phi, y=r \sin \theta \sin \phi$, $z=r \cos \theta$, and by employing the gradient operator in Cartesian coordinates as $\nabla=[\partial / \partial x, \partial / \partial y, \partial / \partial z]^{\mathrm{T}}$. Therefore, the force $\tilde{\mathbf{F}}$ generated by the coil expressed in the coil reference frame $\left[X_{c}, Y_{c}, Z_{c}\right]$ depicted in Fig. 2 can be calculated using the Lorentz force law as

$$
\tilde{\mathbf{F}}=\int_{R_{a}}^{R_{b}} \int_{\theta_{a}}^{\theta_{b}} \int_{-\pi}^{\pi} \mathbf{J} \times \tilde{R}^{\mathrm{T}} \mathbf{B}_{4}\left(x^{\prime}, y^{\prime}, z^{\prime}\right) r^{2} \sin \theta d \phi d \theta d r
$$

where

$$
\left[x^{\prime}, y^{\prime}, z^{\prime}\right]^{\mathrm{T}}=\tilde{R}[\sin \theta \cos \phi, \sin \theta \sin \phi, \cos \theta]^{\mathrm{T}} .
$$

The current density in the coil reference frame is expressed by

$$
\mathbf{J}=J\left[\begin{array}{lll}
-\sin \phi, & \cos \phi, & 0
\end{array}\right]^{\mathrm{T}}
$$

where $J$ is the amplitude of the vector field. The magnetic flux density for any orientation is calculated using (27). Therefore, from (29), we obtain the force generated by a coil and by the

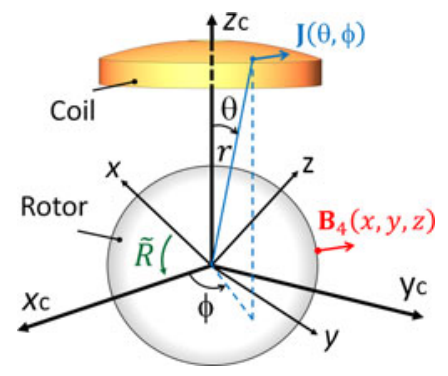

Fig. 2. Stator and coil reference frames for force and torque calculation.

spherical harmonic of degree 3 and order $m$ as

$$
\tilde{\mathbf{F}}^{m}=\int_{R_{a}}^{R_{b}} \int_{\theta_{a}}^{\theta_{b}} \int_{-\pi}^{\pi} \mathbf{J} \times \tilde{R}^{\mathrm{T}} \mathbf{B}_{4}^{m}\left(x^{\prime}, y^{\prime}, z^{\prime}\right) r^{2} \sin \theta d \phi d \theta d r .
$$

Then, the net force generated by a single coil is obtained by summing forces generated by each spherical harmonic as

$$
\tilde{\mathbf{F}}=\sum_{m=-3}^{3} c_{3}^{m} \tilde{\mathbf{F}}^{m}
$$

Finally, notice that the integral (32) can be expressed in closed form using a program of symbolic computation.

\section{B. Torque Generated by a Coil}

Following the same development as for the calculation of the force, the torque $\tilde{\mathbf{T}}$ generated by a coil is obtained by making the cross product of the application points of the infinitesimal forces by their values

$$
\tilde{\mathbf{T}}=\int_{R_{a}}^{R_{b}} \int_{\theta_{a}}^{\theta_{b}} \int_{-\pi}^{\pi} \mathbf{r} \times \mathbf{J} \times \tilde{R}^{\mathrm{T}} \mathbf{B}_{4}\left(x^{\prime}, y^{\prime}, z^{\prime}\right) r^{2} \sin \theta d \phi d \theta d r
$$

where

$$
\mathbf{r}=\left[\begin{array}{lll}
r \sin \theta \cos \phi, & r \sin \theta \sin \phi, \quad r \cos \theta
\end{array}\right]^{\mathrm{T}} .
$$

Then, the torque generated by a single coil and by the spherical harmonic of order $m$ becomes

$\tilde{\mathbf{T}}^{m}=\int_{R_{a}}^{R_{b}} \int_{\theta_{a}}^{\theta_{b}} \int_{-\pi}^{\pi} \mathbf{r} \times \mathbf{J} \times \tilde{R}^{\mathrm{T}} \mathbf{B}_{4}^{m}\left(x^{\prime}, y^{\prime}, z^{\prime}\right) r^{2} \sin \theta d \phi d \theta d r$.

Finally, the net torque provided by the coil is calculated summing the contribution of each spherical harmonic as

$$
\tilde{\mathbf{T}}=\sum_{m=-3}^{3} c_{3}^{m} \tilde{\mathbf{T}}^{m}
$$

\section{Complete Force and Torque Model}

Given a set of 20-coil coordinates $\mathbf{P}_{k}$, each having a current density norm $J_{k}$, the force $\tilde{\mathbf{F}}_{k}$ and torque $\tilde{\mathbf{T}}_{k}$ generated by a coil can be computed using (33) and (37), respectively. Summing forces and torques given by each individual coil in the stator 
frame $[X, Y, Z]$ results in the complete force and torque models

$$
\mathbf{F}=\sum_{k=1}^{20} \tilde{R}_{k} \tilde{\mathbf{F}}_{k}\left(J_{k}\right)=\sum_{m=-3}^{3} c_{3}^{m} \sum_{k=1}^{20} \tilde{R}_{k} \tilde{\mathbf{F}}_{k}^{m}\left(J_{k}\right)
$$

and

$$
\mathbf{T}=\sum_{k=1}^{20} \tilde{R}_{k} \tilde{\mathbf{T}}_{k}\left(J_{k}\right)=\sum_{m=-3}^{3} c_{3}^{m} \sum_{k=1}^{20} \tilde{R}_{k} \tilde{\mathbf{T}}_{k}^{m}\left(J_{k}\right)
$$

where $\tilde{R}_{k}$ shall satisfy

$$
\tilde{R}_{k}^{\mathrm{T}} \mathbf{P}_{k}=\left[\begin{array}{lll}
0, & 0, & 1
\end{array}\right]^{\mathrm{T}} .
$$

By applying the current density norm substitution

$$
J_{k}=\frac{2 N_{t}}{\left(R_{b}^{2}-R_{a}^{2}\right)\left(\theta_{b}-\theta_{a}\right)}
$$

where $N_{t}$ is the number of turns in each coil, (38) and (39) can be written as

$$
\mathbf{F}=\sum_{m=-3}^{3} c_{3}^{m} K_{F}^{m} \mathbf{i} \quad \text { and } \quad \mathbf{T}=\sum_{m=-3}^{3} c_{3}^{m} K_{T}^{m} \mathbf{i}
$$

where $\mathbf{i}$ is a current vector, and $K_{F}^{m}$ and $K_{T}^{m}$ are, respectively, force and torque matrices for the harmonic $m$ and are defined as

$$
K_{F}^{m}=\sum_{k=1}^{20} \tilde{R}_{k} \tilde{\mathbf{F}}_{k}^{m}\left(J_{k}\right) \quad \text { and } \quad K_{T}^{m}=\sum_{k=1}^{20} \tilde{R}_{k} \tilde{\mathbf{T}}_{k}^{m}\left(J_{k}\right) .
$$

We emphasize that in (42), forces and torques are expressed as a linear combination of forces and torques provided by each spherical harmonic of order $m$, with the coefficients $c_{3}^{m}$ delivering to the models all the necessary information relative to the orientation of the rotor. Notice that the matrices $K_{F}^{m}$ and $K_{T}^{m}$ given by (43) are constant and can be computed offline. Finally, defining force and torque characteristic matrices $K_{F}$ and $K_{T}$ as

$$
K_{F}=\sum_{m=-3}^{3} c_{3}^{m} K_{F}^{m} \quad \text { and } \quad K_{T}=\sum_{m=-3}^{3} c_{3}^{m} K_{T}^{m}
$$

(42) can be rewritten in a compact form as

$$
\mathbf{F}=K_{F} \mathbf{i} \quad \text { and } \quad \mathbf{T}=K_{T} \mathbf{i} .
$$

\section{Force and Torque Inverse Models}

An inverse model that determines a current vector $\mathbf{i}$ for a desired force $\mathbf{F}$ and torque $\mathbf{T}$ can be derived. The difficulty of the inverse model is that there are 20 degrees of freedom for the current and only 6 degrees of freedom for forces and torques. To calculate the desired inverse models, definitions in (45) can be rewritten in matrix form as

$$
\left[\begin{array}{c}
\mathbf{F} \\
\mathbf{T}
\end{array}\right]=\left[\begin{array}{l}
K_{F} \\
K_{T}
\end{array}\right] \mathbf{i} .
$$

Because the stator has 20 poles $\mathbf{P}_{i}, i=1,2, \ldots, 20$, each corresponding to one vertex of a dodecahedron, each pole faces another and one has $\mathbf{P}_{i}=-\mathbf{P}_{20+1-i}, i=1,2, \ldots, 10$. Consequently, due to the symmetry of both the stator coils and the magnetization pattern, $\mathbf{F}_{i}=\mathbf{F}_{20+1-i}, i=1,2, \ldots, 10$ and $\mathbf{T}_{i}=-\mathbf{T}_{20+1-i}, i=1,2, \ldots, 10$, where $\mathbf{F}_{i}$ and $\mathbf{T}_{i}$ are, respectively, the force and the torque produced by coil $i$. Therefore, it can be verified that force and torque characteristic matrices are mutually orthogonal, and hence $K_{F} K_{T}^{\mathrm{T}}=0$. Because of this decoupling characteristic, force and torque can be controlled independently, which is most suitable for the control of the spherical actuator. Hence, applying the right inverse matrix to (46), we obtain the inverse model for the force and torque

$$
\mathbf{i}=M_{F} \mathbf{F}+M_{T} \mathbf{T}
$$

where

$$
M_{F}=K_{F}^{\mathrm{T}}\left(K_{F} K_{F}^{\mathrm{T}}\right)^{-1} \text { and } M_{T}=K_{T}^{\mathrm{T}}\left(K_{T} K_{T}^{\mathrm{T}}\right)^{-1} .
$$

Observe that, because forces and torques generated by the stator span a 3-D space, the rank of the matrices $K_{F}$ and $K_{T}$ is equal to three, and so are the ones of $K_{F} K_{F}^{\mathrm{T}}$ and $K_{T} K_{T}^{\mathrm{T}}$. Therefore, their right inverse always exists. Furthermore, (47) is also the least squares solution of (46) and hence the resulting current vector is the minimal norm solution and the unique vector minimizing the dissipated energy.

\section{Determination of THE Spherical Harmonic COEFFICIENTS FOR FORCE AND TORQUE MODELS}

Seven spherical harmonic decomposition coefficients are necessary to completely characterize the force and torque produced by the rotor in any orientation using (44). In this section, a method based on measuring the radial component of the magnetic flux density at multiple locations is proposed to determine these coefficients. Starting from the magnetic scalar potential (25), the radial component of the magnetic flux density in the airgap can be calculated as

$$
B_{4 r}(r, \theta, \phi)=K_{1} B_{\mathrm{r}} \frac{\partial}{\partial r}\left[r^{-4}\left(R_{4}^{-7} r^{7}-1\right)\right] \sum_{m=-3}^{3} c_{3}^{m} Y_{3}^{m}(\theta, \phi)
$$

from which we derive

$$
\frac{B_{4 r}(r, \theta, \phi)}{K_{1} B_{\mathrm{r}} r^{-5}\left(4+3 R_{4}^{-7} r^{7}\right)}=\sum_{m=-3}^{3} c_{3}^{m} Y_{3}^{m}(\theta, \phi) .
$$

Therefore, the spherical harmonic decomposition coefficients $c_{3}^{m}$ can be calculated by decomposing the quantity on the lefthand side of (50) on the basis of spherical harmonics of degree 3. Several methods are proposed in the literature to tackle this problem. For instance, the coefficients can be calculated by approximating the integration in (71) as presented in [21]. However, this procedure requires an important number of measurements around the rotor resulting in a large computational effort. Nevertheless, notice that for band-limited functions, which is the case here, the sampling theorem allows the decomposition coefficients to be computed exactly from measurements taken on the equiangular latitude-longitude grid [22]. This approach is very attractive as it requires a limited number of measurements but forces the sensors to be placed on the latitude-longitude grid, which is not very practical for the reaction sphere because of the presence of the stator coils. We propose to sample 
the radial component of the magnetic flux density at $N \geq 7$ mutually noncollinear locations equidistant from the rotor center and to solve the decomposition problem using least-squares techniques as originally suggested in [23]. Therefore, suppose that $N$ one-axis magnetic flux density sensors are placed at $\mathbf{S}_{k}=\left(R_{s}, \theta_{k}, \phi_{k}\right), k=1,2, \ldots, N$. Then, defining

$$
B_{4, k}^{\perp}=B_{4 r}\left(R_{s}, \theta_{k}, \phi_{k}\right), \quad \Pi=K_{1} B_{\mathrm{r}} R_{s}^{-5}\left(4+3 R_{4}^{-7} R_{s}^{7}\right)
$$

and decomposing $c_{3}^{m}$ in its real and imaginary parts as

$$
c_{3}^{m}=a_{3}^{m}+i b_{3}^{m}, \quad|m| \leq 3
$$

we can write

$$
\begin{aligned}
& \frac{B_{4, k}^{\perp}}{\Pi}=c_{3}^{0} Y_{3}^{0}(\theta, \phi) \\
& \quad+\sum_{m=1}^{3}\left[c_{3}^{-m} Y_{3}^{-m}\left(\theta_{k}, \phi_{k}\right)+c_{3}^{m} Y_{3}^{m}\left(\theta_{k}, \phi_{k}\right)\right] \\
& =c_{3}^{0} Y_{3}^{0}\left(\theta_{k}, \phi_{k}\right)+\sum_{m=1}^{3}\left[\overline{c_{3}^{m} Y_{3}^{m}\left(\theta_{k}, \phi_{k}\right)}+c_{3}^{m} Y_{3}^{m}\left(\theta_{k}, \phi_{k}\right)\right] \\
& =c_{3}^{0} Y_{3}^{0}\left(\theta_{k}, \phi_{k}\right)+2 \sum_{m=1}^{3} \operatorname{Re}\left\{c_{3}^{m} Y_{3}^{m}\left(\theta_{k}, \phi_{k}\right)\right\} \\
& =\frac{a_{3}^{0}}{2} R_{3}^{0}\left(\theta_{k}, \phi_{k}\right)+\sum_{m=1}^{3} a_{3}^{m} R_{3}^{m}\left(\theta_{k}, \phi_{k}\right)+\sum_{m=1}^{3} b_{3}^{m} I_{3}^{m}\left(\theta_{k}, \phi_{k}\right)
\end{aligned}
$$

where $R_{3}^{m}\left(\theta_{k}, \phi_{k}\right)=2 \operatorname{Re}\left\{Y_{3}^{m}\left(\theta_{k}, \phi_{k}\right)\right\}$ and $I_{3}^{m}\left(\theta_{k}, \phi_{k}\right)=$ $-2 \operatorname{Im}\left\{Y_{3}^{m}\left(\theta_{k}, \phi_{k}\right)\right\}$. Therefore, defining a vector of $N$ magnetic flux measurements

$$
\mathbf{B}_{4}^{\perp}=\left[B_{4,1}^{\perp}, B_{4,2}^{\perp}, \ldots, B_{4, N}^{\perp}\right]^{\mathrm{T}}
$$

and defining the $N \times 7$ matrix $A$ as

$$
A^{\mathrm{T}}=\left[\begin{array}{cccc}
\frac{1}{2} R_{3}^{0}\left(\theta_{1}, \phi_{1}\right) & \frac{1}{2} R_{3}^{0}\left(\theta_{2}, \phi_{2}\right) & \ldots & \frac{1}{2} R_{3}^{0}\left(\theta_{N}, \phi_{N}\right) \\
R_{3}^{1}\left(\theta_{1}, \phi_{1}\right) & R_{3}^{1}\left(\theta_{2}, \phi_{2}\right) & \ldots & R_{3}^{1}\left(\theta_{N}, \phi_{N}\right) \\
R_{3}^{2}\left(\theta_{1}, \phi_{1}\right) & R_{3}^{2}\left(\theta_{2}, \phi_{2}\right) & \ldots & R_{3}^{2}\left(\theta_{N}, \phi_{N}\right) \\
R_{3}^{3}\left(\theta_{1}, \phi_{1}\right) & R_{3}^{3}\left(\theta_{2}, \phi_{2}\right) & \ldots & R_{3}^{3}\left(\theta_{N}, \phi_{N}\right) \\
I_{3}^{1}\left(\theta_{1}, \phi_{1}\right) & I_{3}^{1}\left(\theta_{2}, \phi_{2}\right) & \ldots & I_{3}^{1}\left(\theta_{N}, \phi_{N}\right) \\
I_{3}^{2}\left(\theta_{1}, \phi_{1}\right) & I_{3}^{2}\left(\theta_{2}, \phi_{2}\right) & \ldots & I_{3}^{2}\left(\theta_{N}, \phi_{N}\right) \\
I_{3}^{3}\left(\theta_{1}, \phi_{1}\right) & I_{3}^{3}\left(\theta_{2}, \phi_{2}\right) & \ldots & I_{3}^{3}\left(\theta_{N}, \phi_{N}\right)
\end{array}\right]
$$

the desired coefficients $\mathbf{x}=\left[a_{3}^{0}, a_{3}^{1}, a_{3}^{2}, a_{3}^{3}, b_{3}^{1}, b_{3}^{2}, b_{3}^{3}\right]^{\mathrm{T}}$ can be computed solving $\Pi A \mathbf{x}=\mathbf{B}_{4}^{\perp}$ using the left-inverse matrix of $A$ as

$$
\mathbf{x}=\frac{1}{\Pi}\left(A^{\mathrm{T}} A\right)^{-1} A^{\mathrm{T}} \mathbf{B}_{4}^{\perp}
$$

The matrix $A^{\mathrm{T}} A$ is nonsingular if the sensors are placed so that they are mutually noncollinear. Observe that the matrix

$$
P=\frac{1}{\Pi}\left(A^{\mathrm{T}} A\right)^{-1} A^{\mathrm{T}}
$$

of (56) is constant and can be computed offline. Hence, force and torque characteristics for the rotor in any possible orientation can be determined in closed form by projecting the measurements of the radial component of the magnetic flux density on the column space of $P$. In the next section, we summarize the proposed method and we discuss its implementation procedure.

\section{Summary and ImPlementation Procedure}

The objective of this section is to summarize the proposed technique to compute force and torque models and to highlight the implementation details. In the previous section, we proposed a method to measure the seven necessary spherical harmonic decomposition coefficients $c_{3}^{m}$, with $|m| \leq 3$. Hence, given these coefficients, force and torque characteristic matrices are calculated using (44). However, applying definition (52) and invoking the properties employed to derive (53), the force characteristic matrix $K_{F}$ in (44) can be rewritten as

$$
K_{F}=\frac{a_{3}^{0}}{2} K_{F, \mathrm{R}}^{0}+\sum_{m=1}^{3} a_{3}^{m} K_{F, \mathrm{R}}^{m}+\sum_{m=1}^{3} b_{3}^{m} K_{F, \mathrm{I}}^{m}
$$

where

$$
K_{F, \mathrm{R}}^{m}=2 \operatorname{Re}\left\{K_{F}^{m}\right\} \quad \text { and } \quad K_{F, \mathrm{I}}^{m}=-2 \operatorname{Im}\left\{K_{F}^{m}\right\} .
$$

Therefore, the force characteristic matrix can be calculated directly from (56) as

$$
\begin{array}{r}
K_{F}=\frac{x_{1}}{2} \cdot K_{F, \mathrm{R}}^{0}+x_{2} \cdot K_{F, \mathrm{R}}^{1}+x_{3} \cdot K_{F, \mathrm{R}}^{2}+x_{4} \cdot K_{F, \mathrm{R}}^{3} \\
+x_{5} \cdot K_{F, \mathrm{I}}^{1}+x_{6} \cdot K_{F, \mathrm{I}}^{2}+x_{7} \cdot K_{F, \mathrm{I}}^{3}
\end{array}
$$

where $x_{k}$ is the $k$ th entry of the solution vector $\mathbf{x}$. Similarly, defining

$$
K_{T, \mathrm{R}}^{m}=2 \operatorname{Re}\left\{K_{T}^{m}\right\} \quad \text { and } \quad K_{T, \mathrm{I}}^{m}=-2 \operatorname{Im}\left\{K_{T}^{m}\right\}
$$

the torque characteristic matrix can be expressed as

$$
\begin{aligned}
K_{T}=\frac{x_{1}}{2} \cdot K_{T, \mathrm{R}}^{0}+ & x_{2} \cdot K_{T, \mathrm{R}}^{1}+x_{3} \cdot K_{T, \mathrm{R}}^{2}+x_{4} \cdot K_{T, \mathrm{R}}^{3} \\
& +x_{5} \cdot K_{T, \mathrm{I}}^{1}+x_{6} \cdot K_{T, \mathrm{I}}^{2}+x_{7} \cdot K_{T, \mathrm{I}}^{3} .
\end{aligned}
$$

We emphasize that the matrices $K_{F, \mathrm{R}}^{m}, K_{F, \mathrm{I}}^{m}, K_{T, \mathrm{R}}^{m}$, and $K_{T, \mathrm{I}}^{m}$ are constant and are computed offline.

\section{A. Implementation Procedure}

The implementation procedure to compute force and torque characteristic matrices for the rotor about any possible orientation is summarized by dividing the operations to be performed as offline and online.

The diagram of the procedure to compute the force characteristic matrix is reported in Fig. 3. Although not reported, the diagram for the torque characteristic matrix has exactly the same structure. Therefore, force and torque characteristic matrices $K_{F}$ and $K_{T}$ are expressed as linear combination of the matrices $K_{F, \mathrm{R}}^{m}, K_{F, \mathrm{I}}^{m}, K_{T, \mathrm{R}}^{m}$, and $K_{T, \mathrm{I}}^{m}$ with the decomposition coefficients $x_{1}, x_{2}, \ldots, x_{7}$. The matrices $K_{F, \mathrm{R}}^{m}, K_{F, \mathrm{I}}^{m}, K_{T, \mathrm{R}}^{m}$, and $K_{T, \mathrm{I}}^{m}$ as well as the projection matrix $P$ are constant and are computed offline. Hence, we emphasize that force and torque 


\section{Operations performed offline}

1) Place $N \geq 7$ mutually non-collinear single-axis magnetic flux density sensors equidistant from the rotor surface so that the matrix $A^{\mathrm{T}} A$ in (56) is fullrank. For instance, $N=7$ sensors could be placed at the center of each stator coils $\mathbf{P}_{1}$ to $\mathbf{P}_{7}$.

2) Given the set of selected sensors, compute the spherical harmonic projection matrix $P \in \mathbb{R}^{7 \times N}$ using definition (55) and (57).

3) Compute force and torque characteristic matrices $K_{F, \mathrm{R}}^{m} \in \mathbb{R}^{3 \times 20}, K_{F, \mathrm{I}}^{m} \in \mathbb{R}^{3 \times 20}, K_{T, \mathrm{R}}^{m} \in \mathbb{R}^{3 \times 20}$, and $K_{T, \mathrm{I}}^{m} \in \mathbb{R}^{3 \times 20}$ using expressions (43), with definitions (59) and (61). These matrices can be computed with a program of symbolic computation.

\section{Operations performed online}

1) Measure the radial component of the flux density and combine the $N$ measurements in the vector $\mathbf{B}_{4}^{\perp} \in \mathbb{R}^{N \times 1}$ defined in (54).

2) Compute the decomposition coefficients $\mathbf{x} \in \mathbb{R}^{7 \times 1}$ projecting the measurement vector $\mathbf{B}_{4}^{\perp}$ on the matrix $P$ previously computed offline. Hence, $\mathbf{x}=$ $P \cdot \mathbf{B}_{4}^{\perp}$.

3) Compute force and torque characteristic matrices $K_{F} \in \mathbb{R}^{3 \times 20}$ and $K_{T} \in \mathbb{R}^{3 \times 20}$ as linear combination of matrices $K_{F, \mathrm{R}}^{m}, K_{F, \mathrm{I}}^{m}, K_{T, \mathrm{R}}^{m}$, and $K_{T, \mathrm{I}}^{m}$ and the measured decomposition coefficients $\mathbf{x}$ as expressed in (60) and (62).

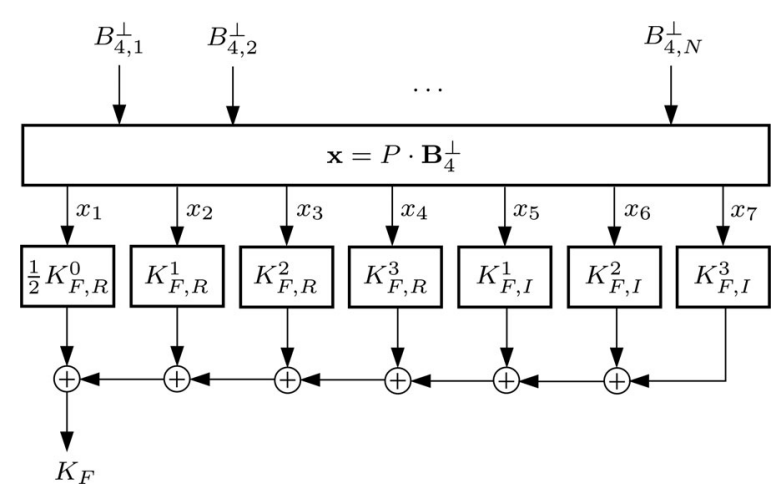

Fig. 3. Diagram for the computation of the force characteristic matrix $K_{F} \in$ $\mathbb{R}^{3 \times 20}$. The matrices $K_{F, \mathrm{R}}^{m}$ and $K_{F, \mathrm{I}}^{m}$ as well as the projection matrix $P$ are constant and are computed offline.

characteristic matrices for the rotor about any possible orientation are computed noniteratively and in a linear fashion. Finally, it should be noted that the magnetic flux density measurements will be affected by the field due to stator currents. However, as illustrated in [5], the measured radial components of the flux density are linear with respect to coil currents. Therefore, the influence of the stator currents on the sensor measurements can be compensated for by using current measurements and the information obtained in a calibration procedure.
TABLE 1

PARAMETERS FOR FINITE ELEMENTS VERIFICATION

\begin{tabular}{|l|l|l|l|}
\hline Inner back iron $R_{1}$ & $75 \mathrm{~mm}$ & Outer coil $R_{b}$ & $99 \mathrm{~mm}$ \\
\hline Back iron $R_{2}$ & $80 \mathrm{~mm}$ & Inner coil angle $\theta_{a}$ & $3.7 \mathrm{deg}$ \\
\hline Magnet $R_{3}$ & $89 \mathrm{~mm}$ & Inner coil angle $\theta_{b}$ & $16 \mathrm{deg}$ \\
\hline Inner stator $R_{4}$ & $99 \mathrm{~mm}$ & Iron permeability $\mu_{r}$ & 1000 \\
\hline Outer stator $R_{5}$ & $103 \mathrm{~mm}$ & Magnet permeability $\mu_{m}$ & 1.1 \\
\hline Inner coil $R_{a}$ & $92 \mathrm{~mm}$ & Magnet remanence $B_{\mathrm{r}}$ & $1.4 \mathrm{~T}$ \\
\hline
\end{tabular}

\section{VERIFICATION With Finite ELEMENT SimUlationS}

\section{A. Simulation Setup}

In this section, magnetic flux density, force, and torque models are verified using finite element simulations. Electromagnetic 3-D finite element simulations are performed using the ac/dc module of COMSOL Multiphysics v4.1 running on a Win7 64bit system equipped with two Intel six-core 3.33-GHz CPUs and 48-GB RAM. Simulation parameters are summarized in Table I. Notice that the verification is performed using a nonferromagnetic stator. Hence, the analytical expression of the magnetic flux density without the metallic stator can be easily obtained from (26) by calculating the limit for the inner stator radius $R_{4} \rightarrow \infty$.

\section{B. Magnetic Flux Density Simulations}

The three components of the magnetic flux density are evaluated about a parametric circular path, which is described in spherical coordinates as $\mathbf{p}=r_{p} \hat{\boldsymbol{r}}+\theta_{p} \hat{\boldsymbol{\theta}}+\phi \hat{\boldsymbol{\phi}}$, where $r_{p}=95.5 \mathrm{~mm}$ and $\phi \in[0,360] \mathrm{deg}$. The evaluation is repeated about four different values of the latitudinal angle $\theta_{p}=\{45,55,65,75\}^{\circ}$. Therefore, for an immobile rotor with a nonferromagnetic stator, the expected radial component of the flux density about these parametric circular paths can be calculated from (25) as

$$
\begin{aligned}
B_{4 r}\left(r_{\mathrm{p}}, \theta_{\mathrm{p}}, \phi\right)= & \lim _{R_{4} \rightarrow \infty}\left\{\left.K_{1} B_{\mathrm{r}} \frac{\partial}{\partial r}\left[r^{-4}\left(R_{4}^{-7} r^{7}-1\right)\right]\right|_{r=r_{\mathrm{p}}}\right\} \\
& \cdot \sum_{m=-3}^{3} c_{3, \mathrm{imm}}^{m} Y_{3}^{m}\left(\theta_{\mathrm{p}}, \phi\right) \\
= & \lim _{R_{4} \rightarrow \infty}\left\{K_{1}\right\} \frac{6 \sqrt{3} B_{\mathrm{r}}}{r_{\mathrm{p}}^{5}} \sin ^{2} \theta_{\mathrm{p}} \cos \theta_{\mathrm{p}} \sin 2 \phi .
\end{aligned}
$$

The tangential components $B_{4 \theta}$ and $B_{4 \phi}$ can be derived in a similar way. Simulated values of the magnetic flux density are compared to the analytical model in Fig. 4. As one can notice, all the three components of the simulated flux density are in strong accordance with the analytical expression of the model.

\section{Force and Torque Simulations}

The objective of this section is to illustrate the ability of the developed force and torque analytical models to produce suitable current vectors to satisfy reference forces and torques for four randomly generated orientations of the rotor. For illustrative purposes, ten single-axis magnetic flux density sensors are 

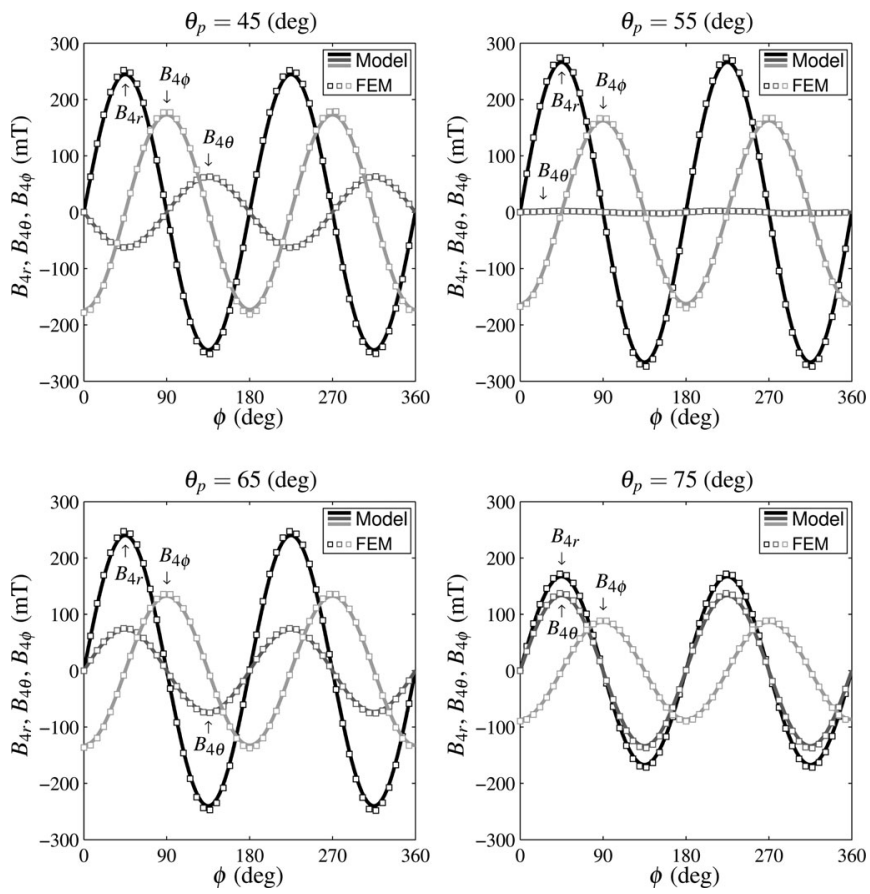

Fig. 4. Simulated magnetic flux density at various values of the angle $\theta$ compared to analytical model.

placed at the center of each stator coil $\mathbf{P}_{1}$ to $\mathbf{P}_{10}$. Hence, the projection matrix $P$ as well as force and torque characteristics matrices $K_{F, \mathrm{R}}^{m}, K_{F, \mathrm{I}}^{m}, K_{T, \mathrm{R}}^{m}$, and $K_{T, \mathrm{I}}^{m}$ are computed as presented in the offline procedure in Section V-A. Subsequently, the simulated magnetic flux density is used to compute matrices $K_{F}$ and $K_{T}$ as described in the online procedure. Then, for each of the four orientations, force and torque characteristic matrices are employed to compute a suitable current vector by applying the inverse model (47) to satisfy three randomly generated pairs of reference forces and torques. Forces and torques are chosen with random direction but with norm equal to $25 \mathrm{~N}$ and $1 \mathrm{~N} \cdot \mathrm{m}$, respectively. Therefore, force and torque inverse models are verified for a total of 12 configurations. Simulations are performed by applying to the stator coils the current vector generated with the inverse model. Finally, simulated forces and torques are computed using the Lorentz integral in COMSOL Multiphysics and compared to the expected reference values. The three components of the reference and simulated forces for the studied configurations are reported in Fig. 5. As can be observed, forces resulting from the proposed simulated model are in agreement with the reference values. Although not reported in this paper, torque simulations resulted in a similar performance.

\section{EXPERIMENTAL VERIFICATION WITH LABORATORY PROTOTYPE}

\section{A. Developed Laboratory Prototype}

A laboratory prototype of the reaction sphere has been developed. The prototype is composed of a permanent magnet rotor and a plastic stator with coils as reported in Figs. 6 and 7, respectively. Notice that manufacturing permanent magnets with
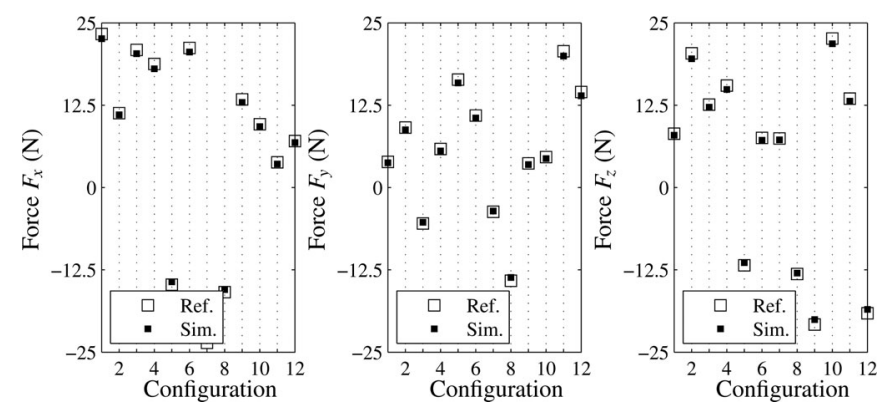

Fig. 5. Reference and simulated forces for multiple randomly selected configurations of the reaction sphere rotor.

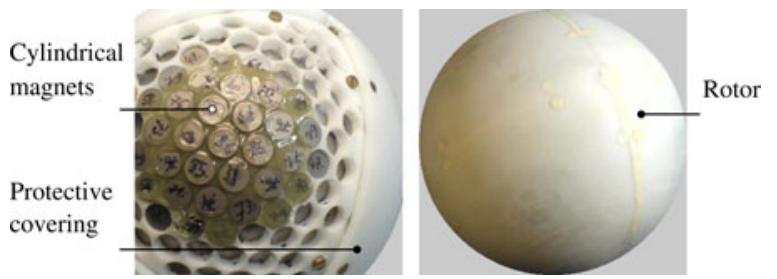

Fig. 6. Rotor during assembly showing cylindrical permanent magnets with (left) different heights. (Right) Final rotor.

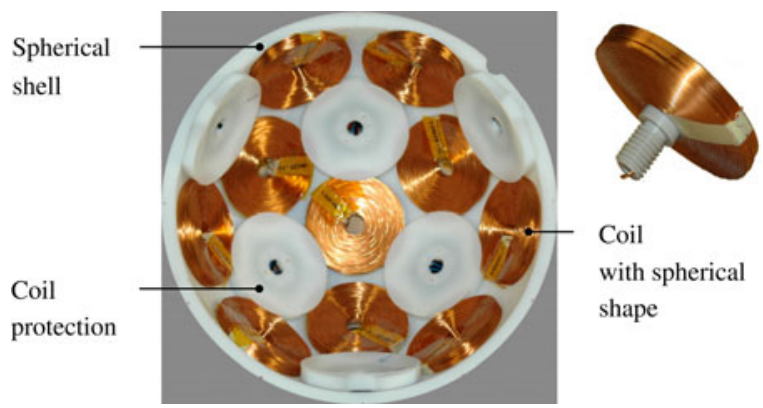

Fig. 7. One of the two plastic stator hemispheres with air-cored coils and plastic protections.

the spherical shape depicted in Fig. 1 to obtain the theoretical remanence $\mathbf{B}_{\mathrm{r}, \mathrm{imm}}$ of (2) is both technologically challenging and expensive. Therefore, in order to obtain a valid approximation of the desired magnetic remanence, the spherical permanent magnet has been discretized using a mosaic of 728 cylindrical magnets glued on the surface of the reaction sphere rotor back iron. These permanent magnets have the same magnetic remanence $B_{\mathrm{r}}$ and their height is varied to approximate the desired spherical harmonic of (2). The stator is manufactured using a nonferromagnetic plastic material that serves as support for the 20 air-cored coils. Notice that the coils have been manufactured with the desired spherical shape. Specifications of this prototype are consistent with Table I.

\section{B. Magnetic Flux Density Measurement}

1) Experimental Setup: The experimental setup for the magnetic flux density measurement is depicted in Fig. 8. The magnetic flux density is measured with a Gaussmeter (MAGNETPHYSIK FH55) equipped with an axial Hall probe (HS-AGB5) that can be placed at multiple declination angles $\theta$ and at 


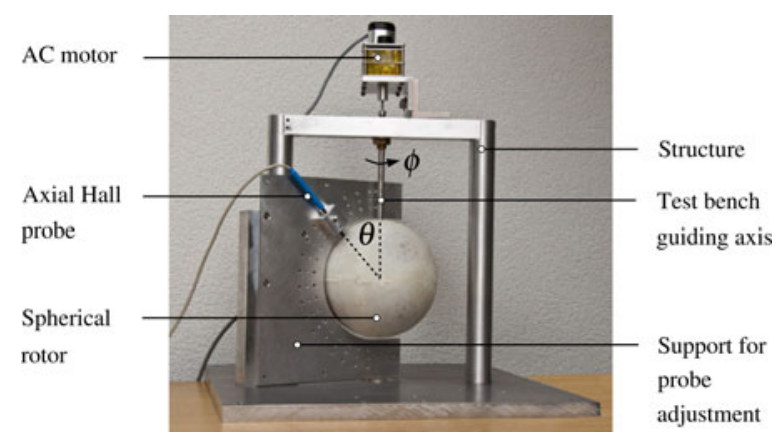

Fig. 8. Experimental setup for magnetic flux density measurement.

various distances from the rotor surface. The axial probe can be positioned to measure the radial component of the magnetic flux density $B_{4 r}$ and both tangential components $B_{4 \theta}$ and $B_{4 \phi}$, which all contribute to generate the Lorentz force. An electric ac motor connected to a rigid guiding axis is employed to rotate the rotor at $0.1 \mathrm{r} / \mathrm{min}$. A dSPACE platform records the measured data at sampling intervals of $0.5 \mathrm{~s}$.

2) Measurement Results: The three components of the measured magnetic flux density at $r=95.5 \mathrm{~mm}$ for different values of angle $\theta$ are compared to the analytical model in Fig. 9. As can be observed, both the radial and tangential components of the measured flux density have the desired sinusoidal profile for all angles $\theta$. However, the amplitudes of all three components of the measured flux density profiles are approximately $20 \%$ weaker than their analytical values due to the discretization of the spherical permanent magnet. As a matter of fact, the discretization is obtained with a mosaic of 728 cylindrical magnets that do not cover the whole rotor surface. Subsequently, the radial components of the magnetic flux density measured about a pole $\left(\theta \approx 55^{\circ}\right.$ and $\left.\phi=45^{\circ}\right)$, from a radius $R_{a}=92 \mathrm{~mm}$ (inner coil radius) to $R_{b}=99 \mathrm{~mm}$ (outer coil radius and location of sensors), are compared to the analytical model in Fig. 10. As one can notice, the ratio $\Delta_{K}$ between the measured and analytical values does not vary significantly throughout the airgap, and it is approximately equal to 0.8 .

3) Discussion: On the basis of the measurements performed, it is assumed that the magnetic flux density $\mathbf{B}_{4}^{\mathrm{p}}$ within the airgap of the developed prototype can be expressed as

$$
\mathbf{B}_{4}^{\mathrm{p}}=\Delta_{K} \mathbf{B}_{4}
$$

where $\mathbf{B}_{4}$ is the flux density with the ideal spherical permanent magnet in (26) while $\Delta_{K}=0.8$ a scalar to account for its discretization. The value of $\Delta_{K}$ is determined experimentally and depends on both the discretization method and the dimension of the rotor.

For open-loop operation, with assumption (64) and invoking linearity, force and torque analytical models proposed in Section III can be easily scaled to account for the discretization by premultiplying $K_{F}$ and $K_{T}$ in (44) with $\Delta_{K}$. Therefore, for the same current vector, the amplitude of forces and torques produced by the developed prototype will be lower by a factor $\Delta_{K}$ than those obtained with the ideal spherical permanent magnet. We emphasize, however, that since $\Delta_{K}$ is a scalar,
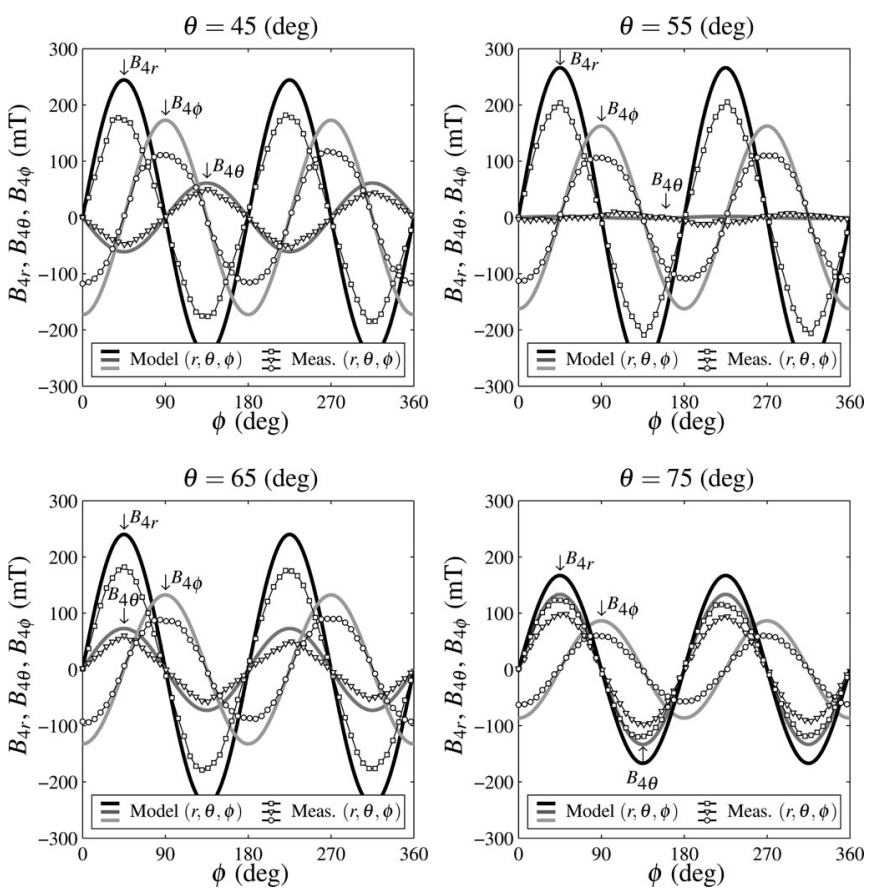

Fig. 9. Measured magnetic flux density at various angles $\theta$ compared to analytical model.

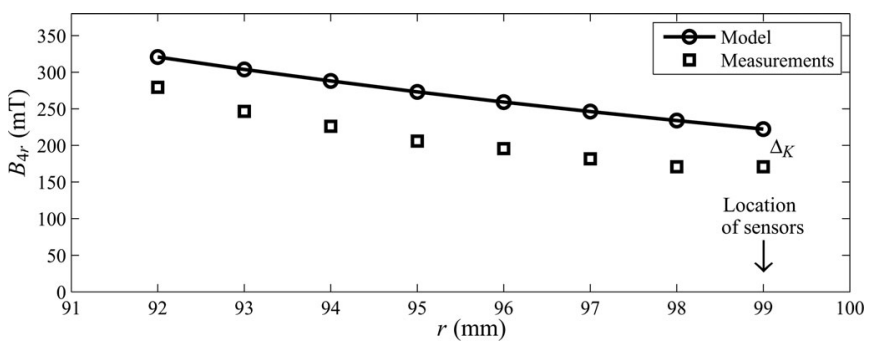

Fig. 10. Measured radial component of the magnetic flux density at various values of the radius $r$ compared to analytical model.

the directions of forces and torques remain virtually unaffected. Vice versa, with the scaled force and torque models, the currents necessary to satisfy a pair of reference force and torque will be higher by a factor $1 / \Delta_{K}$ than those necessary with the ideal spherical magnet. Therefore, the current vector has a larger magnitude to compensate for the weaker flux density due to the discretization of the magnet.

For closed-loop operation, however, rescaling the force and torque characteristic matrices in (43) and the normalization factor $\Pi$ in (51) is possible but not necessary. As a matter of fact, the matrices $K_{F}$ and $K_{T}$ are computed using (60) and (62), where the coefficients $x_{k}$, estimated using (56) from measurements embedded in the vector $\mathbf{B}_{4}^{\perp}$, completely specify the weaker magnetic flux density within the airgap. Therefore, the currents computed to satisfy a pair of reference force and torque using the inverse model (47) will automatically and in a closedloop fashion compensate for the weaker flux density due to the discretization of the magnet. 


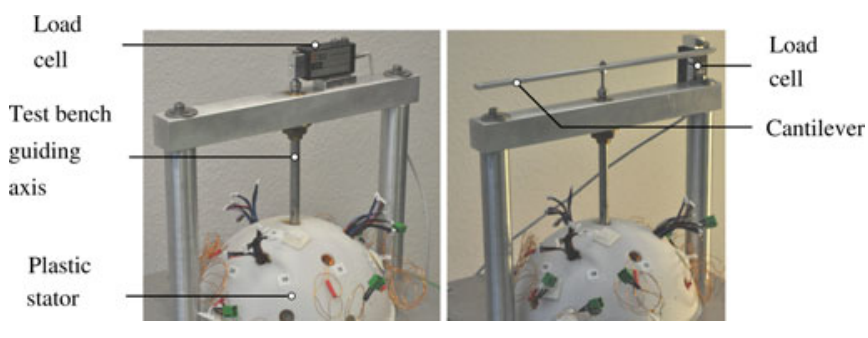

Fig. 11. Experimental setup for (left) force and (right) torque measurement.

\section{Force and Torque Measurement}

1) Experimental Setup: The experimental setup for force and torque measurements is reported in Fig. 11. The sphere is maintained centered inside the stator by a temporary rigid guiding axis to allow the measurement of forces and torques. Only force and torque components parallel to the axis will be measured. We emphasize, however, that this test axis is purely arbitrary and that the procedure for obtaining force and torque models is applied as presented in the previous sections without a priori information on the choice of the rotation axis. For force measurements, the rotor is suspended to a load cell (HMB Typ Z8) that continuously measures the rotor weight. The permanent magnet rotor is mounted inside a plastic stator that also supports the coils. To measure the torque, the rotor is suspended and the torque is measured with a load cell through a cantilever of length $d$ equal to $14.3 \mathrm{~cm}$.

The measurement procedure consists in illustrating the ability of the developed force and torque analytical models to produce suitable current vectors to obtain the desired forces and torques for the rotor about various orientations. For each orientation, described by a rotation of $\alpha$ about the axis, the magnetic flux density is measured at the center of each stator coil $\mathbf{P}_{1}$ to $\mathbf{P}_{9}$ at $R_{s}=99 \mathrm{~mm}$ and the online procedure in Section V-A is followed to compute $K_{F}$ and $K_{T}$. For each orientation, a set of currents $\mathbf{i}_{F}$ and $\mathbf{i}_{T}$ is computed using the inverse model to produce vertical forces of $9.81 \mathrm{~N}$ and torques of $0.4 \mathrm{~N} \cdot \mathrm{m}$ as

$$
\begin{aligned}
& \mathbf{i}_{F}=K_{F}^{\mathrm{T}}\left(K_{F} K_{F}^{\mathrm{T}}\right)^{-1} \cdot\left[\begin{array}{lll}
0, & 0, & 9.81 \cdot 1
\end{array}\right]^{\mathrm{T}} k \\
& \mathbf{i}_{T}=K_{T}^{\mathrm{T}}\left(K_{T} K_{T}^{\mathrm{T}}\right)^{-1} \cdot\left[\begin{array}{lll}
0, & 0, & 0.4
\end{array}\right]^{\mathrm{T}} k
\end{aligned}
$$

where $k$ is a scalar factor, which will be varied to verify the linearity.

2) Measurement Results: Measured forces and torques for different values of the proportionality factor $k$ and for the rotor about different orientations are reported in Fig. 12. Measured values confirm the linearity between stator currents and forces and torques. Moreover, forces and torques obtained about various orientations of the rotor are in good agreement with the analytical models. Differences between the experimental values and the analytical model are mainly caused by fabrication and measurement errors as well as residual features due to the discretization of the magnet.

The currents necessary to obtain the measured forces when the proportionality factor $k$ is equal to 3 are presented in Fig. 13. As discussed in Section III-D, because two facing coils $\mathbf{P}_{i}$ and $\mathbf{P}_{20+1-i}$ produce the same force, one has $\mathbf{i}_{F, i}=\mathbf{i}_{F, 20+1-i}, i=$

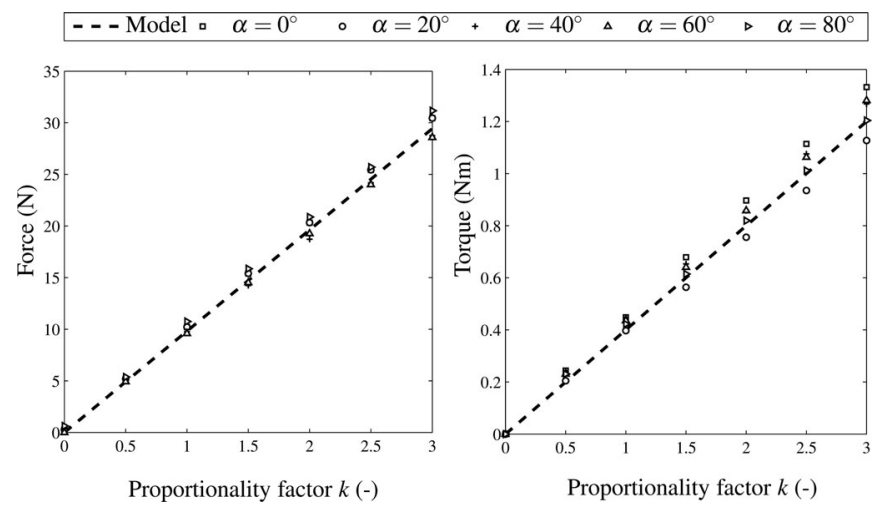

Fig. 12. Measured forces (left) and torques (right) for different orientations of the rotor and different magnitudes of the current vector.

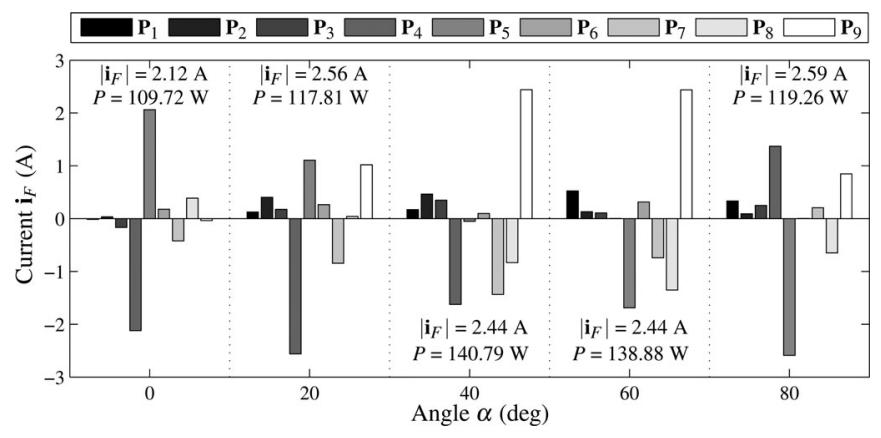

Fig. 13. Half-stator currents $\mathbf{i}_{F}$ for different orientations of the rotor with force proportionality factor $k=3$.

$1,2, \ldots, 10$. Therefore, since coil $\mathbf{P}_{10}$ and $\mathbf{P}_{11}$ are not used in this experiment, only current from $\mathbf{P}_{1}$ to $\mathbf{P}_{9}$ are reported in Fig. 13. For each angle $\alpha$, Fig. 13 indicates the maximum current intensity defined by the vector infinity-norm as $\left|\mathbf{i}_{F}\right|=\max \left|i_{i}\right|, i=1,2, \ldots, 10$, and the total electric power $P$ relative to all 20 stator currents. As can be noticed, the current of each coil depends on the orientation of the rotor described by $\alpha$. Moreover, although the current vector $\mathbf{i}_{F}$ is computed to minimize the dissipated energy for each orientation $\alpha$ using (48), the total electric power varies among the orientations indicating the existence of preferred configurations. Although not reported, similar results are observed for the current $\mathbf{i}_{T}$ necessary to produce the torques reported in Fig. 12. For $k=3$, the current intensity lies between $\left|\mathbf{i}_{T}\right|=1.30 \mathrm{~A}$ and $\left|\mathbf{i}_{T}\right|=1.52 \mathrm{~A}$ while the necessary electric power ranges from $P=52.95 \mathrm{~W}$ to $P=104.00 \mathrm{~W}$. Finally, note that higher forces and torques can be easily generated provided that sufficient electric power can be supplied to the stator coils.

\section{CONCLUSION}

We have presented force and torque analytical models of an innovative spherical actuator. The magnetization of the permanent magnet rotor allows exploiting powerful properties of spherical harmonics under rotation to produce an expression of the magnetic flux density for the rotor in any possible orientation. The resulting model is linear and is parameterized using seven spherical harmonic coefficients that convey the necessary 
information relative to the rotor configuration. We have then proposed an estimation method of the seven spherical harmonic coefficients. This method is based on measuring the radial component of the magnetic flux density at $N \geq 7$ locations equidistant from the rotor surface. Unlike the estimation of the rotor orientation using Euler angles, the proposed technique is linear and is expressed in closed form. Despite being intimately related to the magnetization of the rotor, involving in our case spherical harmonics of degree 3 only, this methodology could also be generalized to take undesired distortions into account, by considering their expansion on harmonic functions of higher degrees in (8). Subsequently, we have summarized the implementation procedure for the online computation of force and torque models, highlighting the attractive computational structure, in which several ingredients are computed offline. Finally, force and torque models are verified with numerical finite element simulations and with the developed laboratory prototype. Although not considered in this paper, the influence of sensor noise and the distortion of the magnetic flux density on the spherical actuator performance will be the topic of our future investigations.

\section{APPENDIX}

\section{SPHERICAL HARMONICS}

In this appendix, a synthetic overview of the definition and relevant properties of spherical harmonics are presented according to [18]. For any integer $n \geq 0$ and integer $m$, with $|m| \leq n$, the complex-valued spherical harmonic of degree $n$ and order $m \geq 0$ is defined in spherical coordinates $(\theta, \phi)$ as

$$
Y_{n}^{m}(\theta, \phi)=\sqrt{\frac{(2 n+1)(n-m) !}{4 \pi(n+m)}} P_{n}^{m}(\cos \theta) e^{i m \phi}
$$

and for $m<0$ as

$$
Y_{n}^{-m}(\theta, \phi)=(-1)^{m} \overline{Y_{n}^{m}(\theta, \phi)}
$$

where $P_{n}^{m}(\cos \theta)$ are associated Legendre functions and $Y_{n}^{m}(\theta, \phi)$ is the complex conjugate of $Y_{n}^{m}(\theta, \phi)$. The spherical harmonics $Y_{n}^{m}(\theta, \phi)$ are orthogonal in $L^{2}\left(S^{2}\right)$

$$
\int_{0}^{\pi} \int_{0}^{2 \pi} Y_{n}^{m}(\theta, \phi) \overline{Y_{k}^{j}(\theta, \phi)} \sin \theta d \theta d \phi=\delta_{n m} \delta_{k j} .
$$

Therefore, any function $f \in L^{2}\left(S^{2}\right)$ can be expanded as

$$
f(\theta, \phi)=\sum_{n=0}^{L} \sum_{m=-n}^{n} c_{n}^{m} Y_{n}^{m}(\theta, \phi)
$$

where the coefficients $c_{n}^{m}$ of expansions are calculated invoking the orthogonality property as

$$
c_{n}^{m}=\int_{0}^{2 \pi} \int_{0}^{\pi} f(\theta, \phi) \overline{Y_{n}^{m}(\theta, \phi)} \sin \theta d \theta d \phi .
$$

Finally, applying a rotation operator on the function $f(\theta, \phi)$ gives some new function $\breve{f}(\theta, \phi)$ whose expansion coefficients are calculated as

$$
\breve{c}_{n}^{m}=\sum_{m^{\prime}} c_{n}^{m^{\prime}} D_{m m^{\prime}}^{n}(\alpha \beta \gamma)
$$

where $D_{m m^{\prime}}^{n}(\alpha \beta \gamma)$ are unitary rotation matrices due to Wigner, which are expressed in terms of the Euler angles $(\alpha \beta \gamma)$.

\section{REFERENCES}

[1] W. J. Larson and J. R. Wertz, Eds., Space Mission Analysis and Design, 3rd ed.. Torrance, CA: Microcosm, Inc., 1999.

[2] M. J. Sidi, Spacecraft Dynamics and Control: A Practical Engineering Approach. Cambridge, U.K.: Cambridge Univ. Press, 2000.

[3] O. Chételat, "Torquer apparatus," U.S. Patent 2010007 303, Jan. 14, 2010

[4] L. Yan, I.-M. Chen, C. K. Lim, G. Yang, and K.-M. Lee, "Modeling and iron-effect analysis on magnetic field and torque output of electromagnetic spherical actuators with iron stator," IEEE/ASME Trans. Mechatronics, [Online]. Available: http://ieeexplore.ieee.org, DOI: 10.1109/TMECH.2011.2159238.

[5] W. Wang, J. Wang, G. W. Jewell, and D. Howe, "Design and control of a novel spherical permanent magnet actuator with three degrees of freedom," IEEE/ASME Trans. Mechatronics, vol. 8, no. 4, pp. 61-71, Dec. 2003.

[6] K. Kahlen, I. Voss, C. Priebe, and R. W. Doncker, "Torque control of a spherical machine with variable pole pitch," IEEE Trans. Power Electron., vol. 19, no. 7, pp. 1628-1634, Nov. 2004.

[7] L. Yan, I.-M. Chen, G. Yang, and K.-M. Lee, “Analytical and experimental investigation on the magnetic field and torque of a permanent magnet spherical actuator," IEEE/ASME Trans. Mechatronics, vol. 11, no. 4, pp. 409-419, Aug. 2006.

[8] B. Dehez, G. Galary, and B. Raucent, "Development of a spherical induction motor with two degrees of freedom," IEEE Trans. Magn., vol. 42 , no. 8, pp. 2077-2088, Aug. 2006.

[9] L. Yan, I.-M. Chen, H. Son, C. K. Lim, and G. Yang, "Analysis of pole configurations of permanent-magnet spherical actuators," IEEE/ASME Trans. Mechatronics, vol. 15, no. 6, pp. 985-989, Dec. 2010.

[10] K.-M. Lee, K. Bai, and J. Lim, "Dipole models for forward/inverse torque computation of a spherical motor," IEEE/ASME Trans. Mechatronics, vol. 14, no. 1, pp. 46-54, Feb. 2009.

[11] L. Yan, I.-M. Chen, C. K. Lim, G. Yang, and K.-M. Lee, Design, Modeling and Experiments of 3-DOF Electromagnetic Spherical Actuators. Reading, MA: Springer-Verlag, 2011.

[12] L. Rossini, O. Chételat, E. Onillon, and Y. Perriard, "Analytical and experimental investigation on the force and torque of a reaction sphere for satellite attitude control," in Proc. IEEE/ASME Int. Conf. Adv. Intell. Mechatron., Budapest, Hungary, 2011, pp. 487-492.

[13] D. Stein, E. R. Scheinerman, and G. S. Chirikjian, "Mathematical models of binary spherical-motion encoders," IEEE/ASME Trans. Mechatronics, vol. 8, no. 2, pp. 234-244, Jun. 2003.

[14] L. Yan, I.-M. Chen, Z. Guo, Y. Lang, and Y. Li, "A three degree-offreedom optical orientation measurement method for spherical actuator applications," IEEE Trans. Autom. Sci. Eng., vol. 8, no. 2, pp. 319-326, Apr. 2011.

[15] W. Fang and H. Son, "Optimization of measuring magnetic fields for position and orientation tracking," IEEE/ASME Trans. Mechatronics, vol. 16, no. 3, pp. 440-448, Jun. 2011.

[16] S. Foong, K.-M. Lee, and K. Bai, "Magnetic field-based sensing method for spherical joint," in Proc. IEEE Int. Conf. Robot. Autom., Anchorage, AK, 2010, pp. 5447-5452.

[17] L. Hu, K.-M. Lee, and X. Fu, "A method based on measured boundary conditions for reconstructing the magnetic field distribution of an electromagnetic mechatronic system," IEEE/ASME Trans. Mechatronics, vol. 15, no. 4, pp. 595-602, Aug. 2010.

[18] D. W. Ritchie and G. J. L. Kemp, "Fast computation, rotation, and comparison of low resolution spherical harmonic molecular surfaces," J. Comput. Chem., vol. 20, no. 4, pp. 383-395, 1998.

[19] A. Makadia and K. Daniilidis, "Rotation recovery from spherical images without correspondences," IEEE Trans. Pattern Anal. Mach. Intell., vol. 28, no. 7, pp. 1170-1175, Jul. 2006.

[20] E. P. Furlani, Permanent Magnet and Electromechanical Devices: Materials, Analysis and Applications. San Diego, CA: Academic, 2001.

[21] G. Burel and H. Henoco, "Determination of the orientation of $3 \mathrm{~d}$ objects using spherical harmonics," Graph. Models Image Process., vol. 57, no. 5, pp. 400-408, 1995.

[22] D. Healy, Jr., D. Rockmore, P. J. Kostelec, and S. S. B. Moore, "FFTs for the 2-sphere-Improvements and variations," J. Four. Anal. Appl., vol. 9, pp. 341-385, 1996.

[23] C. Brechbühler, G. Gerig, and O. Kübler, "Parametrization of closed surfaces for 3-d shape description," Comput. Vis. Image Understand., vol. 61, no. 4, pp. 154-170, 1995. 


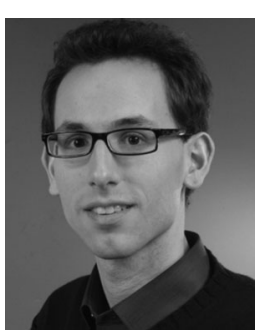

Leopoldo Rossini received the B.Sc. degree in electrical engineering from the University of Applied Sciences of Southern Switzerland, Manno, Switzerland, and the M.Sc. degree in electrical and computer engineering from Purdue University, West Lafayette, IN. $\mathrm{He}$ is currently working toward the Ph.D. degree at the Swiss Federal Institute of Technology, Lausanne, Switzerland.

$\mathrm{He}$ is also with the Swiss Center for Electronics and Microtechnology, Neuchâtel, Switzerland.

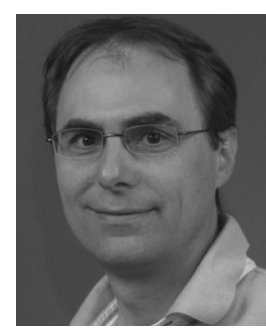

Olivier Chételat received the $\mathrm{Ph} . \mathrm{D}$. degree in robotics from the Swiss Federal Institute of Technology, Lausanne, Switzerland, in 1997.

He joined the Swiss Center for Electronics and Microtechnology, Neuchâtel, Switzerland, in 2001, where he has played an active role as a control expert on several projects, including high-precision mechanisms for optical delay lines. Since 2009, he has been the Head of the Sensing and Control section. He proposed (patent filed in 2007) the reaction sphere configuration with an 8-pole permanent-magnet ro-

tor and a 20-coil stator.

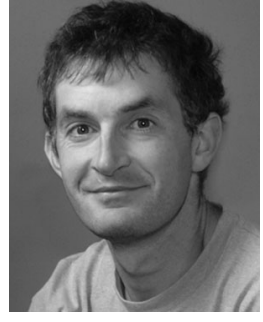

Emmanuel Onillon received the M.Sc. degree in electrical engineering in 1991 and the Ph.D. degree in control and signal processing in 1994 from Supelec, Paris, France.

He joined the Systems Division of the Swiss Center for Electronics and Microtechnology, Neuchâtel, Switzerland, in 2000. He has extensive experience in hardware/software, system integration, and testing as well as multidisciplinary project management in international environment practice, including spacerelated projects.

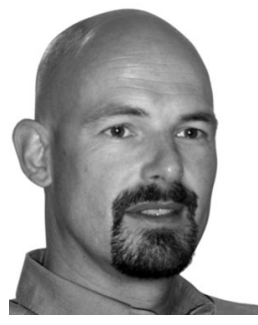

electronic devices.
Yves Perriard was born in Lausanne, Switzerland, in 1965. He received the M.Sc. degree in microengineering and the Ph.D. degree from the Swiss Federal Institute of Technology (EPFL), Lausanne, Switzerland, in 1989 and 1992, respectively.

In 1998, he became a Senior Lecturer and a Professor in 2003 at EPFL, where he is currently the Director of the Integrated Actuator Laboratory. He is also the Vice-Director of the Microengineering Institute, Neuchâtel, Switzerland. His research interests include new actuator designs and associated 